\title{
Charged multiplicity density and number of participant nucleons in relativistic nuclear collisions
}

\author{
Ben-Hao Sa ${ }^{1,4,5,6}$, A. Bonasera ${ }^{2}, \mathrm{Xu} \mathrm{Cai}^{5,4}, \mathrm{An}^{\mathrm{Tai}}{ }^{3}$, and Dai-Mei Zhou \\ 1 China Institute of Atomic Energy, \\ P. O. Box 275 (18), Beijing, 102413 China \\ 2 Laboratorio Nazionale del Sud, Instituto Nazionale Di Fisica Nucleare, \\ v. S. Sofia 4495132 Catania, Italy \\ 3 Department of Physics and Astronomy, University of California, \\ at Los Angeles, Los Angeles, CA 90095 USA \\ 4 Institute of Particle Physics, Huazhong Normal University, Wuhan, 430079 China \\ ${ }^{5}$ CCAST (World Lab.), P. O. Box 8730 Beijing, 100080 China \\ ${ }^{6}$ Institute of Theoretical Physics, Academia Sinica, Beijing, 100080 China *
}

\begin{abstract}
The energy and centrality dependences of charged particle pseudorapidity density in relativistic nuclear collisions were studied using a hadron and string cascade model, JPCIAE. Both the relativistic $p+\bar{p}$ experimental data and the PHOBOS and PHENIX $A u+A u$ data at RHIC energy could be fairly reproduced within the framework of JPCIAE model and without retuning the model parameters. The predictions for $P b+P b$ collisions at the LHC energy were also given. We computed the participant nucleon distributions using different methods. It was found that the number of participant nucleons is not a well defined variable both experimentally and theoretically. Thus it may be inappropriate to use the charged particle pseudorapidity density per participant pair as a function of the number of participant nucleons for distinguishing various theoretical models. A discussion for the effect of different definitions in nuclear radius (diffused or sharp) was given.
\end{abstract}

PACS numbers: 25.75.Dw, 24.10.Lx, 24.85.+p

*Email: sabh@iris.ciae.ac.cn; bonasera@lns.infn.it 


\section{INTRODUCTION}

The main focus of the Relativistic Heavy-Ion Collider (RHIC) at the Brookhaven National Laboratory $(\mathrm{BNL})$ is to explore the phase transition related to the quark deconfinement and the chiral symmetry restoration. The first available experimental data were the energy dependence of charged particle pseudorapidity density in central $A u+A u$ collisions at $\sqrt{s}_{n n}=56$ and $130 \mathrm{GeV}$ from the PHOBOS collaboration [1]. Soon later, the PHENIX collaboration published their data of centrality dependence of the charged particle pseudorapidity density in $A u+A u$ collisions at $\sqrt{s}_{n n}=130 \mathrm{GeV}$ [2] Recently the charged particle pseudorapidity density in central $A u+A u$ collisions at $\sqrt{s}_{n n}=200 \mathrm{GeV}$ was also reported by PHOBOS collaboration [3]. The BRAHMS collaboration published more recently their results of charged particle density from $\mathrm{Au}+\mathrm{Au}$ collisions at $\sqrt{s}_{n n}=130 \mathrm{GeV}$ 世, 5.
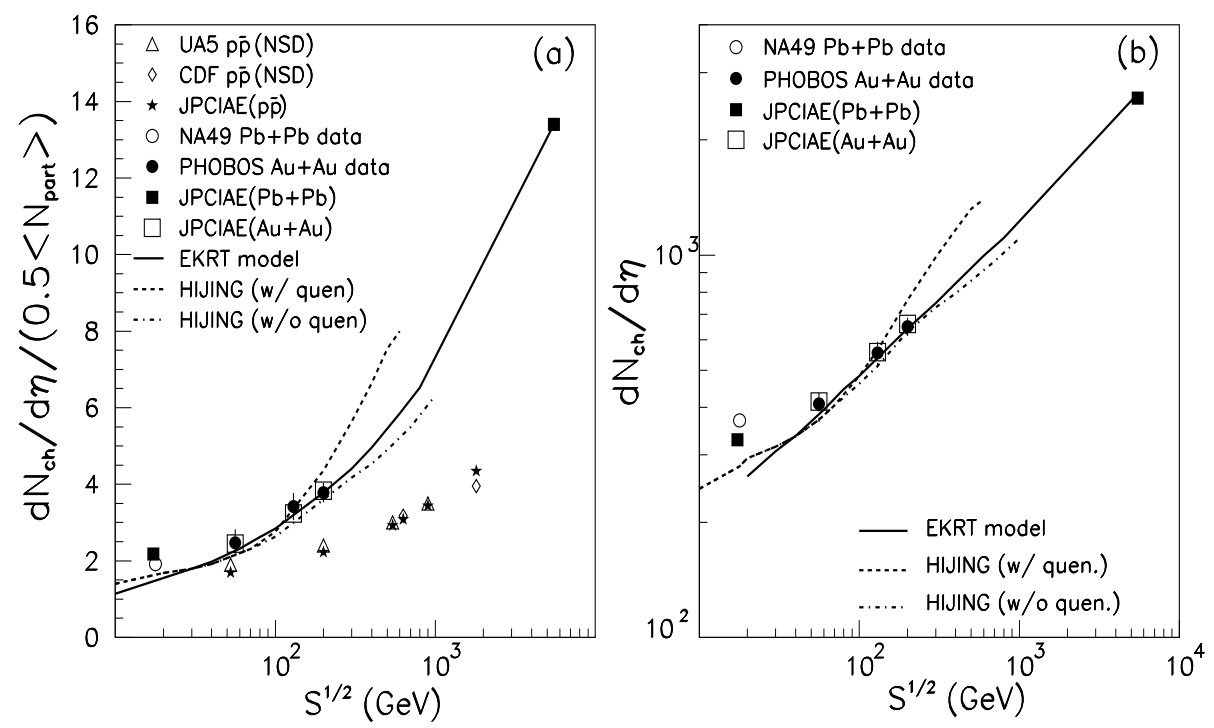

FIG. 1: The energy dependence of the charged particle pseudorapidity density at midpseudorapidity in relativistic $p+\bar{p}$ and central $A+A$ collisions.

It has been predicted that the rare high charged multiplicity in the final state of relativistic nucleus-nucleus collisions might indicate the formation of the Quark-Gluon-Plasma (QGP) phase in the early stage of collisions [6, 7, 8]. In Ref. [9], the centrality dependence of the charged multiplicity has been further proposed to provide information on the relative importance of soft versus hard processes in particle production and therefore to provide a 
means of distinguishing various theoretical models.

The pQCD calculation with assumption of gluon saturation [10, 11] (referred to as the EKRT model later) was first used to study the centrality dependence of the charged particle pseudorapidity density at RHIC. The conventional eikonal approach and the high density QCD (referred to as the KN model later) [12 were also used to investigate the centrality dependence and these two methods surprisingly obtained almost identical centrality dependence. Recently, authors in [13] reported their study of the same issue from the Dual Parton Model. It was found that the experimental observation, the charged particle pseudorapidity density per participant pair slightly increasing with the number of participant nucleons, was reproduced by [9, 12, 13], but contradicted the results of [11].

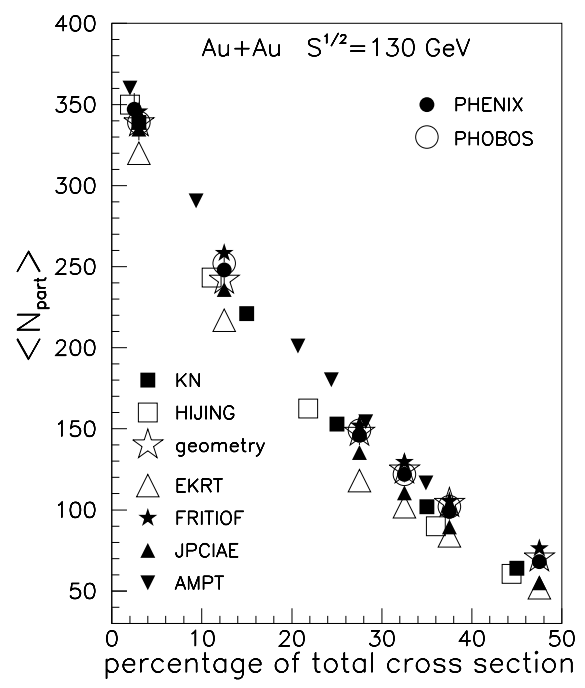

FIG. 2: The number of participant nucleons $\left\langle N_{\text {part }}>\right.$ as a function of the percentage of total cross section.

In this paper a hadron and string cascade model, JPCIAE [14], was employed to study this issue further. Within the framework of this model the experimentally measured energy dependence of the charged particle mid-pseudorapidity density per participant pair both in relativistic $p+\bar{p}$ and $A u+A u$ collisions at RHIC energies was reproduced fairly well without retuning the model parameters. The predictions for the $P b+P b$ collisions at LHC energy were also given. Both the PHENIX [2] and the PHOBOS [15 observations that the charged particle mid-pseudorapidity density per participant pair slightly increases with the number of participant nucleons could be reproduced fairly well by JPCIAE. In 
studying centrality dependence the focus was put on the uncertainties in the definitions and calculations of the number of participant nucleons. However, this study indicated that it is not suitable to use the charged particle mid- pseudorapidity density per participant pair as a function of the number of participant nucleons to constrain theoretical models for particle production, because the number of participant nucleons is not a well defined physical variable both experimentally and theoretically. The effect of the different definitions in nuclear radius (diffused or sharp) on the number of participant nucleons, the charged particle midpseudorapidity density, and the charged particle mid-pseudorapidity density per participant pair as a function of the number of participant nucleons was discussed. A brief version of the part contents of this paper has been published elsewhere [16].

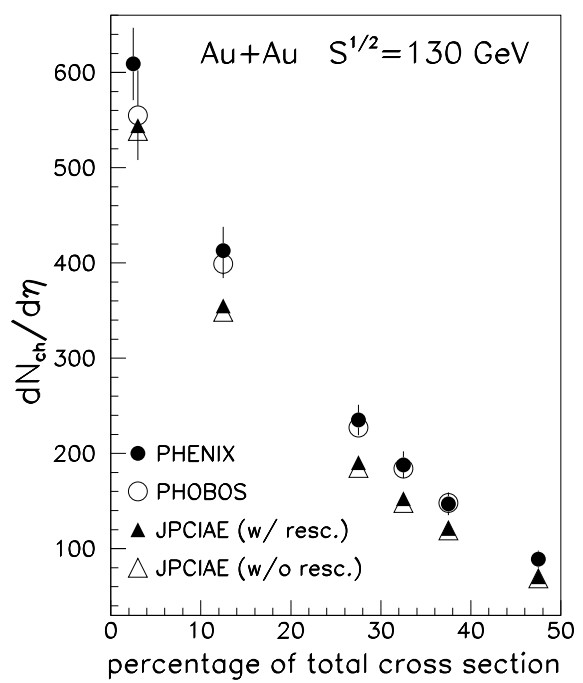

FIG. 3: The charged particle pseudorapidity density at mid-pseudorapidity in $A u+A u$ collisions at $\sqrt{s}_{n n}=130 \mathrm{GeV}$ as a function of the percentage of total cross section.

\section{MODELS}

The JPCIAE model was developed based on PYTHIA [17], which is a well known event generator for hadron-hadron collisions. In the JPCIAE model the radial position of a nucleon in colliding nucleus $A$ (indicating the atomic number of this nucleus as well) is sampled 
randomly according to the Woods-Saxon distribution

$$
\rho(r) \sim \frac{\rho_{0}}{1+\exp \left(\left(r-R_{A}\right) / \alpha\right)}
$$

where $\rho_{0}$ refers to the normal nuclear density, $\alpha \sim 0.54 \mathrm{fm}$ stands for the nuclear diffusion edge, and $R_{A}$ is the nuclear radius of nucleus $A$. The solid angle of the nucleon is sampled uniformly in $4 \pi$. Each nucleon is given a beam momentum in z direction and zero initial momenta in $\mathrm{x}$ and $\mathrm{y}$ directions. The collision time of each colliding pair is calculated under the requirement that the least approach distance of the colliding pair along their straight line trajectory (mean field potential is not taken into account in JPCIAE) should be smaller than $\sqrt{\sigma_{t o t} / \pi}$. Here $\sigma_{t o t}$ refers to the total cross section. The nucleon-nucleon collision with the least collision time is then selected from the initial collision list to perform the first collision. Both the particle list and the collision list are then updated such that the new collision list may consist of not only nucleon- nucleon collisions but also collisions between nucleons and produced particles and between produced particles themselves. The next collision is selected from the new collision list and the processes above are repeated until the collision list is empty.
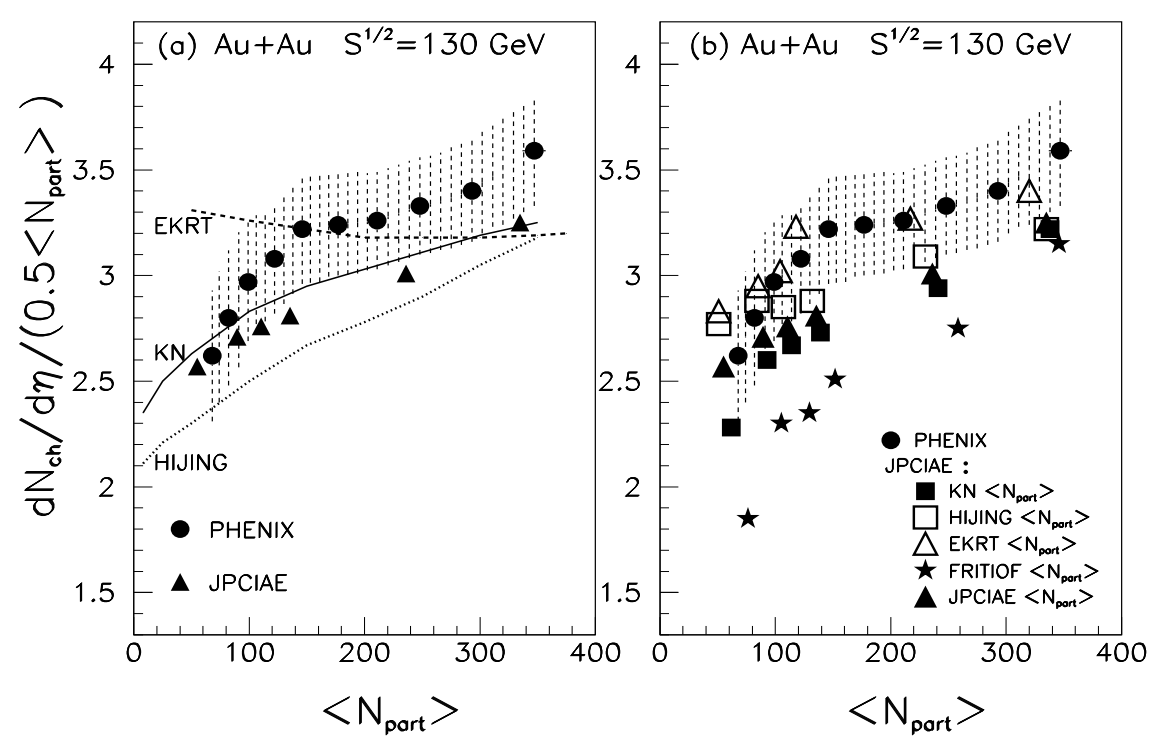

FIG. 4: The charged particle pseudorapidity density per participant pair at mid-pseudorapidity in $A u+A u$ collisions at $\sqrt{s}_{n n}=130 \mathrm{GeV}$ as a function of the number of participant nucleons, $<N_{\text {part }}>$.

For each collision pair, if its CMS energy is larger than a given cut, we assume that 
strings are formed after the collision and PYTHIA is used to deal with particle production. Otherwise, the collision is treated as a two-body collision [18, [9, 20]. The cut $(=4 \mathrm{GeV}$ in the program) was chosen by observing that JPCIAE correctly reproduces charged multiplicity distributions in $A A$ collisions [14]. An important feature of JPCIAE at relativistic energies is that QCD parton-parton scatterings are included through PYTHIA [21], which causes charged particle yields to increase with collision energy as well as centrality since minijet production rates increase with energy and number of collisions suffered by participant nucleons. It should be noted that the JPCIAE model is not a simple superposition of nucleonnucleon collisions since the rescattering among participant nucleons, spectator nucleons, and produced particles is taken into account. We refer to [14 for more about the JPCIAE model.

Since the number of participant nucleons, $N_{\text {part }}$, plays a crucial role in the presentation of PHOBOS or PHENIX data we first make a study on $N_{\text {part }}$. As the direct measurement of $N_{\text {part }}$ is not available, in the fixed target experiments the number of participant nucleons from the projectile nucleus with atomic number $A$, for instance, is estimated by

$$
N_{\text {part }}^{p}=A *\left(1-\frac{E_{Z D C}}{E_{\text {beam }}^{\text {kin }}}\right),
$$

where $E_{Z D C}$ refers to the energy deposited in the Zero Degree Calorimeter (ZDC), dominated by the energy deposition from the projectile spectator nucleons, and $E_{b e a m}^{k i n}$ is the kinetic energy of beam [22]. However, in the collider experiments, in order to obtain $N_{\text {part }}$ one has to relate the measurements to the Monte Carlo simulations. In PHENIX, for instance, simulations for the response of the Beam-Beam Counter and the ZDC were used to calculate $N_{\text {part }}$ via the Glauber model [2]. In PHOBOS, $N_{\text {part }}$ is derived by relating HIJING simulations to the signals in the paddle counter [15]. Therefore, $N_{\text {part }}$ here is a modeldependent variable.

Theoretically, the number of participant nucleons in a collision of $A+B$ at impact parameter $b$ can be estimated in different ways:

1. In the geometry method [23] $N_{\text {part }}(b)$ reads

$$
\begin{array}{r}
N_{\text {part }}(b)=N_{\text {part }}^{A}(b)+N_{\text {part }}^{B}(b), \\
N_{\text {part }}^{A}(b)=\rho_{A} \int d V \theta\left(R_{A}-\left(x^{2}+(b-y)^{2}+z^{2}\right)^{1 / 2}\right) \theta\left(R_{B}-\left(x^{2}+y^{2}\right)^{1 / 2}\right), \\
N_{\text {part }}^{B}(b)=\rho_{B} \int d V \theta\left(R_{B}-\left(x^{2}+y^{2}+z^{2}\right)^{1 / 2}\right) \theta\left(R_{A}-\left(x^{2}+(b-y)^{2}\right)^{1 / 2}\right),
\end{array}
$$




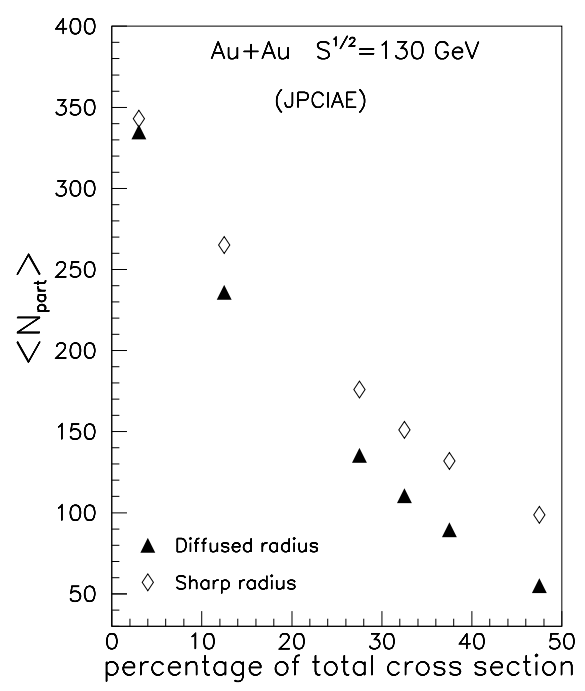

FIG. 5: The number of participant nucleons $\left\langle N_{\text {part }}>\right.$ as a function of the percentage of total cross section, triangles: diffused nuclear radius, rhombuses: sharp nuclear radius.

where $\theta(x)=0$ if $x<0$ and $\theta(x)=1$ otherwise, $\rho_{A}\left(\rho_{B}\right)$ is the nuclear density of the projectile (target) nucleus and the nuclear density is normalized to the atomic number.

2. In the Glauber model, $N_{\text {part }}(b)$ is calculated through

$$
N_{\text {part }}(b)=\int d^{2} s T_{A}(\vec{b}-\vec{s})\left[1-\exp \left(-\sigma_{i n} T_{B}(\vec{s})\right)\right]+\int d^{2} s T_{B}(\vec{s})\left[1-\exp \left(-\sigma_{i n} T_{A}(\vec{b}-\vec{s})\right)\right]
$$

where $\sigma_{\text {in }} \approx 40 \mathrm{mb}$ is the inelastic nucleon-nucleon cross section at RHIC energies and $T_{A}\left(T_{B}\right)$ refers to the nuclear thickness function of nucleus $A(B)$ and is normalized to $A(B)$ 11.

3. In the dynamical simulation of $A+B$ collisions, $N_{\text {part }}(b)$ can be estimated via counting the participant or spectator nucleons and averaging over events simulated at a given impact parameter $b$. However, there are multifarious in simulating models such as: FRITIOF [24], VENUS [25], HIJING [26], JPCIAE [14], UrQMD [27, and AMPT [28], etc. . Not only is the theoretical uncertainty related to the calculation of $N_{\text {part }}(b)$ in each of the above models large but also the definitions of participant nucleons or spectator nucleons are different among each other. We give only a necessary description for the following dynamical simulations mentioned in this paper: 
- In FRITIOF [24 and HIJING [26], the wounded nucleons, i.e., nucleons which suffer at least one inelastic collision, are counted and identified as $N_{\text {part }}(b)$. It should be pointed out here that in FRITIOF and HIJING the produced particles from the string fragmentation do not have rescattering.

- In JPCIAE, we have counted the nucleons involved in at least one inelastic nucleon-nucleon collision with string excitation and identified them as $N_{\text {part }}(b)$.

- In AMPT [29], the spectator nucleons, $N_{\text {spec }}(b)$, are counted in the final state without rescattering and $N_{\text {part }}(b)$ is then calculated through

$$
N_{\text {part }}(b)=(A+B)-N_{\text {spec }}(b) .
$$

The spectator nucleons here refer to the nucleons with zero transverse momentum and beam energy in the final state of the AMPT simulation.

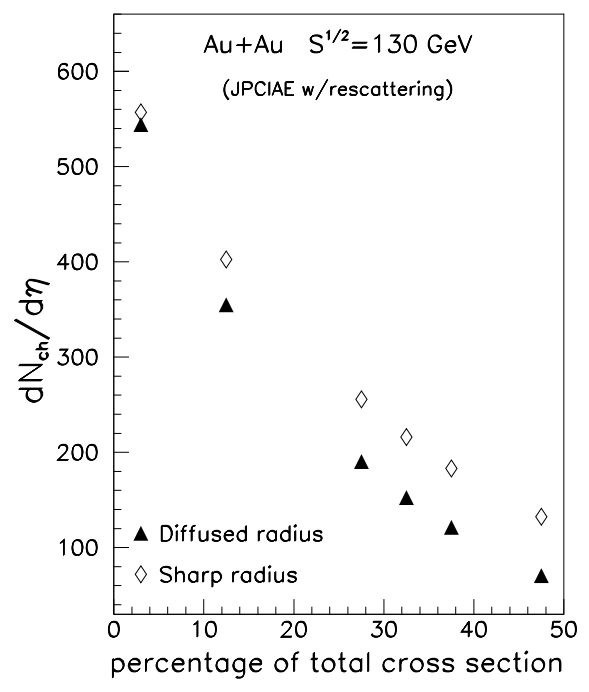

FIG. 6: The charged particle pseudorapidity density at mid-pseudorapidity in $A u+A u$ collisions at $\sqrt{s}_{n n}=130 \mathrm{GeV}$ as a function of the percentage of total cross section, triangles: diffused nuclear radius, rhombuses: sharp nuclear radius.

In the PHENIX or PHOBOS experiment, centrality bin was defined by the cut in the particle multiplicity distribution and expressed as the percentage of geometrical (total) cross section [2], $g$. However, in the theoretical calculation it is more convenient to define the centrality by impact parameter $b$. In order to compare the experimental data of centrality 
dependence with the theoretical results a relation between $g$ and $b$ is required, which is obtained from the definition of the geometrical cross section, that reads

$$
b=\sqrt{g} \times b_{\max }, b_{\max }=R_{A}+R_{B}
$$

where $R_{A}=1.12 A^{1 / 3}+0.54 \mathrm{fm}$, for instance, is the radius of nucleus $A$ with diffused edge. However, $R_{A u}=7.5 \mathrm{fm}$ is taken in our calculations since this value (corresponding to $\sigma_{\text {geo. }}=7.2 \mathrm{~b}$ ) was used in [2] to extract the number of participant nucleons in $A u+A u$ collisions. We give in Tab. 1 the mapping between the centrality bin ( $g$ bin, selected from Tab. 1 in [2]) and the $b$ bin according to Eq. (8), and the averaged impact parameter, $\bar{b}$, over the $b$ bin according to the $b^{2}$ law.

Table 1. The mapping between the $g$ bin and the $b$ bin in $A u+A u$ collisions (case of diffused nuclear radius)

\begin{tabular}{c|c|c}
\hline \hline centrality bin & bin of $b(\mathrm{fm})$ & $\bar{b}(\mathrm{fm})$ \\
\hline below $6^{*}$ & below 3.67 & 2.45 \\
$10-15$ & $4.74-5.81$ & 5.29 \\
$25-30$ & $7.50-8.22$ & 7.87 \\
$30-35$ & $8.22-8.87$ & 8.55 \\
$35-40$ & $8.87-9.49$ & 9.18 \\
$45-50$ & $10.1-10.6$ & 10.4 \\
\hline \hline
\end{tabular}

* below 5 for PHENIX.

As the experimental data were averaged over events in each $g$ bin the theoretical results, to be compared with the experimental data, are also averaged over the corresponding $b$ bin. We denote the number of participant nucleons after averaging over $g$ or $b$ bin as $<N_{\text {part }}>$ later.

\section{RESULTS AND DISCUSSIONS}

In Fig. 1(a) the experimental data of charged particle pseudorapidity density per participant pair at mid-pseudorapidity in relativistic $p+\bar{p}$ (open triangles and rhombuses with 
error bar) and in central $A+A$ collisions (open circles with error bar for $P b+P b$ at SPS and full circles with error bar for $A u+A u$ at RHIC) [1, [3] were compared with the results of the JPCIAE model (full stars for $p+\bar{p}$, open squares for $A u+A u$ collisions at RHIC, and full squares for $P b+P b$ at $\sqrt{s}_{n n}=17.3$ and $5500 \mathrm{GeV}$ ). In addition, the results from other models were plotted as follows: the dashed and dotted-dash curves are from the HIJING model [9] with and without jet quenching, respectively, the solid curve are from the EKRT model [1]. The EKRT results were obtained from [9] directly, except that the EKRT result for $A u+A u$ collisions at $\sqrt{s}_{n n}=5500 \mathrm{GeV}$ was taken from [30]. Fig. 1 (b) is the same as (a) but for $A+A$ collisions only and the vertical coordinate here is the charged particle pseudorapidity density itself. One knows from Fig. 1 that both the data of $p+\bar{p}$ and $A+A$ collisions at relativistic energies are also reproduced fairly well by the JPCIAE model.

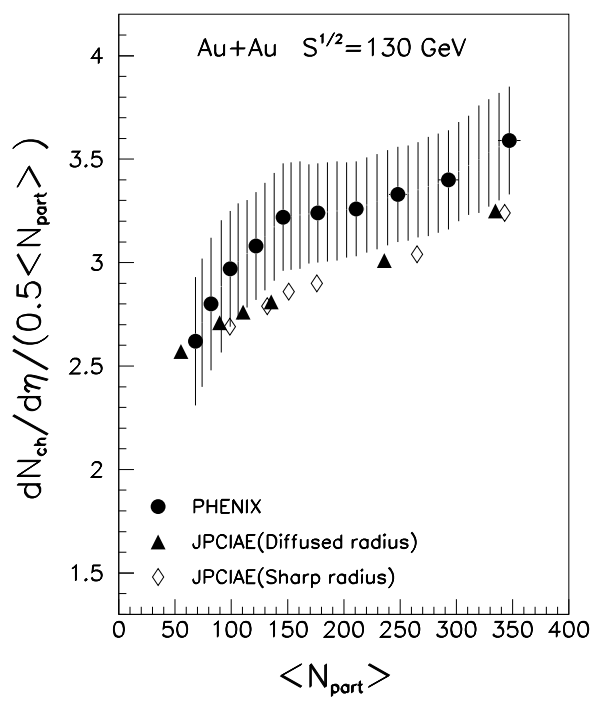

FIG. 7: The charged particle pseudorapidity density per participant pair at mid-pseudorapidity in $A u+A u$ collisions at $\sqrt{s}_{n n}=130 \mathrm{GeV}$ as a function of $\left\langle N_{\text {part }}\right\rangle$. The circles with shaded area of systematic errors: PHENIX data, triangles: JPCIAE (diffused radius), rhombuses: JPCIAE (sharp radius).

In Fig. 2 the $<N_{\text {part }}>$ extracted by PHENIX [2 and PHOBOS 15 from $A u+A u$ collisions at $\sqrt{s}_{n n}=130 \mathrm{GeV}$ were compared with the model computations. The horizontal axis in Fig. 2 is the percentage of geometrical cross section, $g$, and each $g$ bin is represented by its middle point for convenience in plotting (the same for Fig. 3, 5, and 6 ). The solid and open circles with error bar in Fig. 2 were PHENIX and PHOBOS results, respectively. 
$<N_{\text {part }}>$ from the geometry method, open stars, were from the geometric $N_{\text {part }}(b)$ (Eq. 3 - 5) after averaging over $b$ sampled randomly in each $b$ bin due to the $b^{2}$ law. Similarly, the FRITIOF, JPCIAE, and AMPT [29] results, full stars, full triangle-ups, and full triangledowns in Fig. 2, were obtained averaging over events simulated for $b$ sampled randomly in each $b$ bin due to the $b^{2}$ law, respectively. In the EKRT model [11], the curves of $N_{\text {part }}(b)$ vs. $b / R_{A}$ were given and $N_{\text {part }}$ is calculated by the Glauber method with $R_{A}=1.12 A^{1 / 3}$ $0.86 A^{-1 / 3}$. The open triangle-ups in Fig. 2 are the EKRT model results taken from the $\sqrt{s}_{n n}=130 \mathrm{GeV}$ one of those curves according to the $\bar{b}$ in Tab. 1. Originally, HIJING $N_{\text {part }}$ [31] were calculated for individual $b$, the HIJING points, open squares, in Fig. 2 were plotted after relating $b$ to $g$ according to Eq. (8). The full squares in Fig. 2 were the results of the KN model taken from Tab. 1 in [12 under the centrality bins of $0-6,10-20,20-30,30$ - 40, and $40-50 \%$, respectively. In the KN model, $\left\langle N_{\text {part }}\right\rangle$ was the result of $N_{\text {part }}(b)$ from the Glauber model after averaging over centrality bin. In this approach, the particle multiplicity and impact parameter were related by a Gaussian distribution with parameters fixed via fitting the PHOBOS charged multiplicity distribution. As proved in [32], such kind of average is approximately equivalent to the average method based on Eq.(8). From Fig. 2 one knows that the discrepancies among the PHENIX or PHOBOS and the model results are visible.

The charged particle pseudorapidity density at mid-pseudorapidity in $A u+A u$ collisions at $\sqrt{s}_{n n}=130 \mathrm{GeV}$ as a function of the percentage of geometrical cross section was given in Fig. 3. In this figure, the full and open circles with error bar are the PHENIX [2] and PHOBOS [15] data, respectively. The full and open triangles, respectively, are the JPCIAE results with and without rescattering. One knows from Fig. 3 that the rescattering only leads to a few percent increase in the charged multiplicity although rescattering might enhance yields of strangeness, $\Xi^{-}+\overline{\Xi^{-}}$for instance, by a couple of times.

In Fig. 4 (a) we compared the PHENIX data of charged particle mid-pseudorapidity density per participant pair (full circles with shaded area of systematic errors) [2] with the results of the JPCIAE model (full triangles) and the results of other models (obtained from [2] directly): HIJING (the dotted curve), the KN model (the solid curve), and EKRT (the dashed curve). One sees that except EKRT, three other models predict an increase of $\left(d N_{c h} /\left.d \eta\right|_{\eta=0}\right) /\left(0.5<N_{\text {part }}>\right)$ as a function of $<N_{\text {part }}>$ though the theoretical results seem to underestimate the PHENIX data. Such an increase can be understood in JPCIAE as a 
result of increasing hard parton scatterings per participant nucleon. Fig. 4 (b) compared the PHENIX data to the results of single $d N_{c h} /\left.d \eta\right|_{\eta=0}$ from JPCIAE normalized by the $<N_{\text {part }}>$ from different models (taken from the corresponding curve in Fig. 2 at the middle point of $g$ bins for KN, HIJING, and EKRT models): full squares, $<N_{\text {part }}>$ from the KN model, open squares from HIJING, open triangles from EKRT, full stars from FRITIOF, and full triangles from JPCIAE. One sees from Fig. 4 (b) that starting from the charged particle mid-pseudorapidity density obtained in JPCIAE, but using $<N_{\text {part }}>$ from different models, the corresponding results of $\left(d N_{c h} /\left.d \eta\right|_{\eta=0}\right) /\left(0.5<N_{\text {part }}>\right)$ are different visibly among each other, in peripheral collisions especially. Therefore it might be inappropriate using $\left(d N_{c h} /\left.d \eta\right|_{\eta=0}\right) /\left(0.5<N_{\text {part }}>\right)$ as a function of $<N_{\text {part }}>$ to distinguish various theoretical models for particle production since $\left\langle N_{\text {part }}\right\rangle$ is not a well defined physical variable.

If one uses the sharp nuclear radius, $R_{A}=1.12 A^{1 / 3} \mathrm{fm}\left(R_{A u}=6.50 \mathrm{fm}\right)$, instead of diffused one used above, the corresponding mapping between the centrality bin ( $g$ bin) and the $b$ bin from Eq. (8) is given in Tab. 2 . Fig. 5, 6, and 7 give, respectively, the comparisons between the JPCIAE results of diffused nuclear radius and the JPCIAE results of sharp nuclear radius in the $\left\langle N_{\text {part }}>\right.$ as a function of centrality, the $d N_{c h} /\left.d \eta\right|_{\eta=0}$ as a function of centrality, and the $\left(d N_{c h} /\left.d \eta\right|_{\eta=0}\right) /\left(0.5<N_{\text {part }}>\right)$ as a function of $<N_{\text {part }}>$. One knows from Fig. 5 and 6 that there are observable discrepancies between the results of diffused nuclear radius and the results of sharp nuclear radius, in peripheral collisions especially. However, in the

Table 2. The mapping between the $g$ bin and the $b$ bin in $A u+A u$ collisions (case of sharp nuclear radius)

\begin{tabular}{c|c|c}
\hline \hline centrality bin & bin of $b(\mathrm{fm})$ & $\bar{b}(\mathrm{fm})$ \\
\hline below $^{*}$ & below 3.20 & 2.13 \\
$10-15$ & $4.11-5.03$ & 4.59 \\
$25-30$ & $6.50-7.12$ & 6.82 \\
$30-35$ & $7.12-7.69$ & 7.41 \\
$35-40$ & $7.69-8.22$ & 7.96 \\
$45-50$ & $8.72-9.19$ & 8.86 \\
\hline \hline
\end{tabular}

* below 5 for PHENIX. 
$\left(d N_{c h} /\left.d \eta\right|_{\eta=0}\right) /\left(0.5<N_{\text {part }}>\right)$ vs. $<N_{\text {part }}>$ plot both results are close to each other as shown in Fig. 7 . That indicates again that it is hard using $\left(d N_{c h} /\left.d \eta\right|_{\eta=0}\right) /\left(0.5<N_{\text {part }}>\right)$ as a function of $\left\langle N_{\text {part }}\right\rangle$ to distinguish various theoretical models for particle production since $N_{\text {part }}$ is not a well defined variable. As one sees from Fig. 5 and 6 that for most central collision both diffused and sharp nuclear radii work well in mapping centrality bin and $b$ bin therefore we do not redraw a figure as Fig. 1 for sharp nuclear radius. However, it should be pointed out that using sharp nuclear radius in mapping centrality bin and $b$ bin is inconsistent with that the nuclear diffusion edge is introduced in Woods-Saxon distribution initiating the nucleons in nucleus.

\section{CONCLUSIONS}

In summary, we used the hadron and string cascade model, JPCIAE, to investigate the energy and centrality dependences of charged particle pseudorapidity density at midpseudorapidity in relativistic $p+\bar{p}$ and $A+A$ collisions. Both the relativistic $p+\bar{p}$ experimental data and the PHOBOS and PHENIX data of $A u+A u$ collisions at RHIC energies could be reproduced fairly well within the framework of the JPCIAE model without retuning any parameters. The JPCIAE model predictions for $\mathrm{Pb}+\mathrm{Pb}$ collisions at the LHC energy were also given. Both the PHENIX [2] and the PHOBOS [15] observations that the charged particle mid-pseudorapidity density per participant pair slightly increases with the number of participant nucleons could be reproduced fairly well by JPCIAE. This study shows that since $<N_{\text {part }}>$ is not a well defined physical variable both experimentally and theoretically it may be hard to use charged particle pseudorapidity density per participant pair at mid-pseudorapidity as a function of $\left\langle N_{\text {part }}\right\rangle$ to distinguish various theoretical models for particle production. A discussion for the effects of the different definitions in nuclear radius (diffused or sharp) is given and it is indicatd that using diffused nuclear radius might be better.

\section{ACKNOWLEDGMENTS}

Finally, the financial supports from NSFC (19975075, 10135030, and 10075035) in China 
and DOE in USA are acknowledged.

[1] B. B. Back, et al., PHOBOS collab., Phys. Rev. Lett. 85, 3100 (2000).

[2] K. Adcox, et al.,, PHENIX collab., Phys. Rev. Lett. 86, 3500 (2001).

[3] B. B. Back, et al., PHOBOS collab., nucl-ex/0108009.

[4] I. G. Bearden, et al., BRAHMS collab., Phys. Lett. B 523, 227 (2001).

[5] I. G. Bearden, et al., BRAHMS collab., nucl-ex/0112001.

[6] L. van Hove, Phys. Lett. B 118, 138 (1982).

[7] M. I. Gorenstein, S. N. Yang and C. M. Ko, Phys. Lett. B 281, 197 (1992).

[8] J. I. Kapusta and A. P. Vischer, Phys. Rev. C 52, 2725 (1995).

[9] Xin-Nian Wang and M. Gyulassy, Phys. Rev. Lett. 86, 3496 (2001).

[10] K. J. Eskola, K. Kajantie, P.V. Ruuskanen and K. Tuominen, Nucl. Phys. B570, 379 (2000).

[11] K. J. Eskola, K. Kajantie and K. Tuominen, Phys. Lett. B 497, 39 (2001).

[12] D. Kharzeev and M. Nardi, nucl-th/0012025, Phys. Lett. B 507, 121 (2001).

[13] A. Capella and D. Sousa, Phys. Lett. B 511, 185 (2001).

[14] Ben-Hao Sa, An Tai, Hui Wang and Feng-He Liu, Phys. Rev. C 59, 2728 (1999); Ben-Hao Sa and An Tai, Phys. Rev. C 62, 044905 (2000).

[15] B. B. Back, et al., PHOBOS collab., nucl-ex/0105011.

[16] Ben-Hao Sa, A. Bonasera, An Tai, and Dai-Mei Zhou, to appear in Phys. Lett. B .

[17] T. Sjöstrand, Comput. Phys. Commun. 82, 74 (1994).

[18] J. Cugnon, T. Mizutain, and J. Vandermeulen, Nucl. Phys. A 352, 505 (1981).

[19] G. F. Bertsch and S. Das Gupta, Phys. Rep. 160, 189 (1988);

A. Bonasera, F. Gulminelli, and J. Molitoris, Phys. Rep. 243, 1(1994).

[20] An Tai and Ben-Hao Sa, Comput. Phys. Commun. 116, 353 (1999).

[21] T. Sjöstrtand and M. Zijl, Phys. Rev. D36, 2019 (1987).

[22] L. Ahle, et al., E802 Collab., Phys. Rev. C 59, 2173 (1999).

[23] Bi-Xia Miao and Wei-Qin Chao, Nucl. Phys. A494, 620 (1989); Ben-Hao Sa, Yu-Ming Zheng, and Xiao-Ze Zhang, Phys. Rev. C 40, 2680 (1989).

[24] Hong Pi, Z. Phys. C 57, 485 (1993).

[25] K. Werner, Z. Phys. C42, 85 (1989); Phys. Rep. 232, 87 (1993). 
[26] X. N. Wang and M. Gyulassy, Phys. ReV. D44, 3501 (1991); Comput. Phys. Commun. 83, 307 (1994).

[27] S. A. Bass, et al., Prog. Part. Nucl. Phys. 41, 255 (1998); nucl-th/9803035.

[28] Bin Zhang, C. M. Ko, Bao-An Li, and Zi-Wei Lin, Phys. Rev. C61, 067901 (2000); Zi-Wei Lin, S. Pal, C. M. Ko, Bao-An Li, and Bin Zhang, Phys. Rev. C64, 011902 (2001).

[29] Zi-Wei Lin, private communication.

[30] K. J. Eskola, K. Kajantie and K. Tuominen, hep-ph/0106330.

[31] X. N. Wang, private communication.

[32] W. Broniowski and W. Florkowski, nucl-th/0110020, Phys. Rev. C65, 024905 (2002) 